\title{
The relationship between visuospatial ability and perceptual motor function in Parkinson's disease
}

\author{
Marcus Richards, Lucien J Cote, Yaakov Stern
}

\begin{abstract}
To assess the contribution of visuoperceptual function to complex visuomotor responding in Parkinson's disease, 14 patients with idiopathic PD and 12 normal controls matched for age, education and general intellectual function were administered a visual tracing task. No difference was found between the groups on two visuoperceptual tests, the Benton Line Orientation test and a test of trajectory judgement. However, patients were significantly impaired in tracing a sawtooth design when two consecutive angles of the sawtooth were occluded. This impairment occurred in reproducing the basic form of the stimulus and not with accuracy of fine detail. These results suggest higher-order perceptual motor dysfunction independent of any breakdown in basic visuoperceptual processing or loss of fine motor control. It is concluded that Parkinsonian patients are unable to use sensory information accurately to plan and execute complex or new movements.
\end{abstract}

(f Neurol Neurosurg Psychiatry 1993;56:400-406)

In 1978 Flowers $^{1}$ demonstrated that patients with Parkinson's disease (PD) were able to track a target moving across a screen in a ramp or sawtooth pattern, but that tracking performance deteriorated during brief intervals when the target was made to disappear. This impairment was particularly pronounced if target visibility was withdrawn during a change in sweep direction and thus when a movement reversal was required. Flowers argued that this abnormality was not due to loss of fine motor control but arose from a failure to generate and to use predictive strategies.

Stern et $a^{23}$ extended this investigation and showed that PD patients made more errors than controls in tracing a static sawtooth design within sections where a gap obscured a turn in the pathway. This effect was maximal when two consecutive turns were obscured. These authors suggested that this deficit was cognitive in nature and that $P D$ patients were unable to generate or execute an adequate motor plan to guide movements. This hypothesis assumes that such tracing impairments are not caused by perceptual disturbances, but support for this assumption is uncertain. Several studies have shown that PD patients are significantly impaired in judging linear orientation, particularly, visual vertical ${ }^{5}$ and angle, as measured by the Benton Line Orientation test. ${ }^{69}$ The issue is controversial, however, and other investigators have found no difference between patients and controls on this latter test. ${ }^{10-13}$ It is also possible that difficulty in tracing a partially occluded stimulus arises from an inability to forecast the trajectory of the pathway, although PD patients and controls were equally able to judge the putative intersection of a short line and a horizontal baseline. ${ }^{14}$ This was true whether the trajectory was direct or reflected from a rebound surface. Until these negative findings have been replicated, it is fair to state that this question is not resolved.

We therefore administered a sawtooth tracing task to PD patients and controls matched for age and education, in conjunction with the Benton Line Orientation Test and a directional forecast test. In line with Stern's suggestion that PD patients have difficulty in planning and modulating ongoing activity in the absence of external guidance, ${ }^{4}$ we proposed that difficulty with the tracing task would be observed in the absence of impaired visuoperceptual function.

\section{Method}

Subjects

Fourteen patients (9 male, 5 female) with idiopathic PD were recruited during neurological outpatient evaluation and provided informed consent. Mean (SD) age was 69.93 $(7 \cdot 24)$ years and mean (SD) years of education was $15.57(2.53)$. Neurological examination was performed by a senior neurologist (LC). The presence and severity of extrapyramidal signs were rated according to the Columbia University Parkinson's Disease Rating Scale. ${ }^{15}$ Mean (SD) PD duration was $5.98(5.29)$ years, mean Hoehn and Yahr ${ }^{16}$ score was $2.27(0.79)$ (with none greater than 3 ) and the mean Schwab Activities of Daily Living $^{17}$ rating was $76 \cdot 82 \%(21 \cdot 36) \%$. Twelve of these patients were utilising dopamine replacement therapy (levodopa/carbidopa combination) and fourteen were taking a monoamine oxidase B inhibitor. Of the latter, ten were taking selegiline and three were enrolled in a trial for a new drug (R019-6327). One patient was also taking bromocriptine. No patients were taking anticholinergic medication or amantadine at the time of testing. Patients with a history or clin- 
ical evidence of stroke, other neurodegenerative disease or frank visual problems (for example, field cut or diplopia) were excluded. All patients scored less than 2 on the Mentation item of the Columbia University Parkinson's Disease Rating Scale; a score of 2 or more indicates dementia. ${ }^{18}$

The control group consisted of twelve normal volunteers recruited from the local community and matched with the PD patients for age and education. Mean age for the controls was $72.08(6.34)$ years and mean years of education was $14.08(2 \cdot 19)$. No controls had any neurological condition at the time of testing and none were taking medications which affect the CNS. All patients and controls were right handed. To compare the groups for overall intellectual function, all subjects were administered the modified Mini-Mental Status Examination (MMSE ${ }^{19-21}$ ). Mean total MMSE score was $50 \cdot 21(4 \cdot 17)$ for the PD patients and $50 \cdot 25(4 \cdot 22)$ for the controls.

\section{Materials and procedure}

The following tests were administered to all subjects:

1) The Benton Line Orientation test ${ }^{22}$ was given according to standard instructions, where subjects were required to choose two lines from a multiple array of lines with the same angle and orientation as two target lines.

2) A test of directional forecast was adapted from Della Sala et $a l^{14}$ for use with pencil and paper. During this test, subjects were presented with stimuli in black ink on white paper, secured to a firm surface. The test consisted of two tasks. For Task A, the stimuli consisted of a horizontal baseline $19 \mathrm{~cm}$ long at the bottom of the page, and a $3 \mathrm{~cm}$ line at the top of the page at an angle of either $5,10,15,20$, 25 or 30 degrees from vertical. This shorter line was located near either the left or right upper corner and projected down towards the horizontal baseline. Subjects were required to imagine this short line extending to the bottom of the page and to mark, as accurately as possible with a pencil, its projected intersection with the horizontal baseline. Each $5^{\circ}$ angle was presented on both the left and right side of the paper, so that a total of 12 trials were given. The order of the six angle sizes and their right/left orientation was counterbalanced. For Task B, a similar horizontal baseline was presented near the bottom of the paper. However, a short line, identical in length to the one in Task A, was also located near the bottom and projected to the top of the paper, where a horizontal "rebound" surface was located. Subjects were asked to imagine the short line extending up to the rebound surface, reflecting away at the same angle and returning back to the lower baseline. They were then required to mark this final intersection, as in Task A. Two analogies ("like a beam of light striking a mirror/like a billiard ball bouncing off the side") were given to clarify the task requirement. Twelve trials were administered, as in Task A. Angle size, order of angle and right/left orientation were counterbalanced. For each trial, absolute and relative deviation $(\mathrm{mm})$ from the true intersection was measured. For the latter, the deviation was given a sign according to whether the mark was within the true intersection and the side of the paper closest to the short line (negative), or between the true intersection and the opposite side of the paper (positive). No time limit was imposed for this test.

3) A tracing task, adapted from Stern et $a l^{23}$ for pencil and paper use, was administered. Subjects were required to trace, using a pencil, over a sawtooth design printed in black ink on white paper (see fig 1, Path 1, shown reduced to one-half the size of the original).

All subjects used their dominant hand (all were right handed) and were instructed to trace over the printed line with a pencil as accurately as possible. They were required to trace from right to left, from the beginning to the end of the line and were told not to lift the pencil from the paper, to make the movement as smooth and as continuous as possible and to thus refrain from stopping at any point once the tracing had started. No time-limit was imposed, however. When this tracing was complete, Path 1 was removed and, without previous warning, Path 2 was introduced. This consisted of the same figure, except that one segment (the left upper angle) was deleted. Subjects were given the following instructions: "Here is the same figure that you just traced, but as you can see, a piece has now been removed (examiner indicates by pointing). I want you to trace over the figure as if it were complete, that is, as if it was the same as the first one and that missing piece was not removed." These instructions were elaborated if the subject was unclear about the task, but under no circumstances did the examiner mime the sequence of movements. Just as before, subjects were instructed to trace from right to left, to not lift the pencil from the paper and to make the movement as smooth and as continuous as possible. Finally, and again without prior knowledge, Path 3 was administered. In this path, two contiguous angles were deleted (see fig 2, Path 3). Instructions were as follows: "Here is the same figure once again, but now a bigger piece has been removed. Just like before, I want you to trace over the figure as if it were complete, that is, like it was in the first figure, with nothing removed." Subjects were again reminded about tracing from left to right and not removing the pencil from the paper. Each path was administered once only and always in this same order.

A scoring system was developed, where individual points were awarded for particular features of the tracings. This is summarised in table 1. For Paths 2 and 3, points were scored for tracing the basic form of the figure ("form" points). These points were designed to capture two basic requirements of the task. Because the task assessed the ability to trace the figure in the absence of visual guidance, only angles formed between two freehand lines (that is, lines within an occluded space) 
Path 1

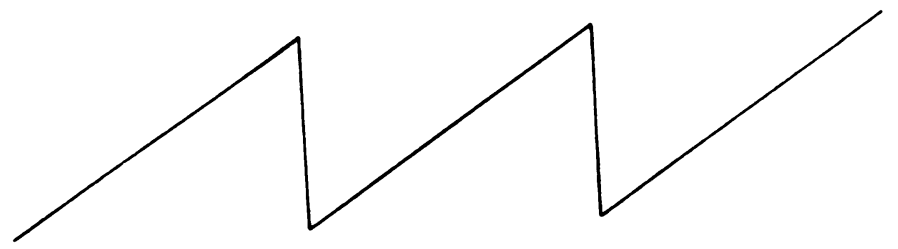

Path 2
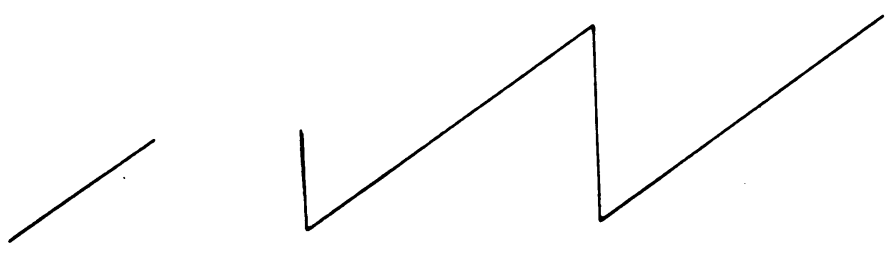

Path 3
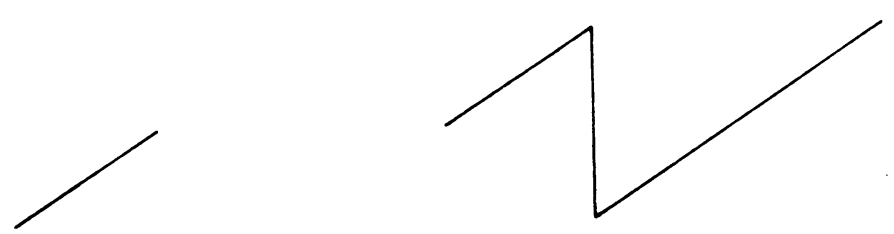

Figure 1 Paths for the sawtooth tracing task.

were considered to be correctly traced (item 1 for Path 2 and items 1 and 3 for Path 3 in table 1). However, Path 3 also assessed the ability to generate a sequence of angles (items 2 and 4 for Path 3 in table 1). For this reason, all angles traced were counted as part of the sequence, even those that were formed between a freehand line and a stimulus line and thus scoring no credit as freehand angles.

As well as these points for reproduction of the basic form of the figure, additional points were awarded for accuracy, in particular, size of the reproduced angles and the proximity of the apex of these angles to their correct location.

Scoring of both the directional forecast task and the sawtooth tracing task was conducted by two independent assistants who were "blind" to group membership.

Data analysis

Before group comparison, normality for all variables was assessed using one-sample Kolmogorov-Smirnov tests (separately for patients and controls). Where no departure from normality was found, between-group analysis of covariance (ANCOVA) was used for single measures and multivariate analysis of covariance (MANCOVA) was used for group comparison of repeated measures. In both cases, age, education and MMSE level were used as covariates, to improve the accuracy of group matching.

\section{Results}

Preliminary $t$ tests showed no statistical difference between patients and controls in age, education or total MMSE score.

Means and standard deviations for the test scores are shown in table 2.

There was no significant difference between groups with the Benton Line Orientation test (ANCOVA ${ }^{125 \text { d.f. }}$. On the Directional Forecast test, there was a steady increase in the magnitude of the deviation as angle size increased. Possible group differences in the trend of these curves were tested, using MANCOVA for polynomial trend analysis, with group as the between-groups factor, absolute deviation for each angle size as the within-subject factor (6 levels) and age, education and MMSE score as covariates. A highly significant angle effect was found for both tasks $\left(T^{2}=3.71, p<0.001\right.$ and $\mathrm{T}^{2}=5.86, \mathrm{p}<0.001$, respectively), at both the linear and quadratic levels. However, neither the group effect nor the group $\mathbf{x}$ angle interaction were significant for these tasks. Results were essentially unchanged when deviation with respect to the left and right of the true target was analysed instead of absolute deviation.

The $F$ ratio for the total tracing score approached significance $(F=3 \cdot 79, p=0.065)$. While the $F$ ratio for Path 2 subtotal was nonsignificant, that for Path 3 was significant $(F=5.00, p=0.036)$, with $P D$ patients achieving a lower score than controls. To investigate the contributions to this difference, form subscores were analysed separately. Only 2 form points were possible for Path 2. One subject from each group lost a single form point when tracing this path. A total of 4 form points were possible for Path 3. However, no subjects scored 0 or 1 or 3 points when tracing this path. Form points for Path 3 were therefore divided into low (poor performance) and high (perfect score). Six patients were located in the low category, compared with only 1 control, whereas all remaining controls (11), but only 8 patients, were located in the high category (Chi square $=3.91, p=0.048)$. These analyses indicate that patients had difficulty in tracing a design, but only when 2 consecutive turns were deleted. Furthermore, poor performance in patients arose specifically from an inability to execute the basic form of the design.

Inspection of the subjects' tracings revealed that patients and controls made qualitatively different form errors during Path 3. The one control who made form errors generated extra angles, whereas all 6 patients who made form errors failed to reproduce at least one missing angle. These are shown in fig 2 (reduced by $65 \%$ of the original size).

The relationship of performance on the 3 visuospatial tests to the MMSE (representing global intellectual function) was then investigated. For PD patients, a moderate positive 
Control

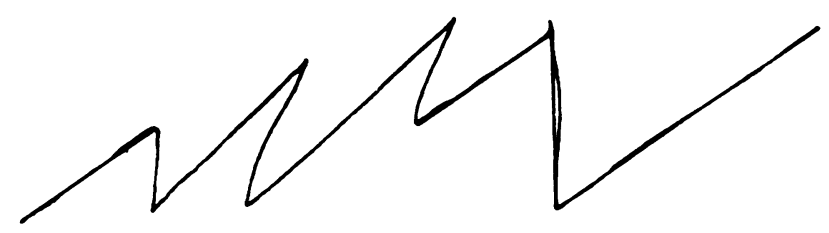

Patient 1

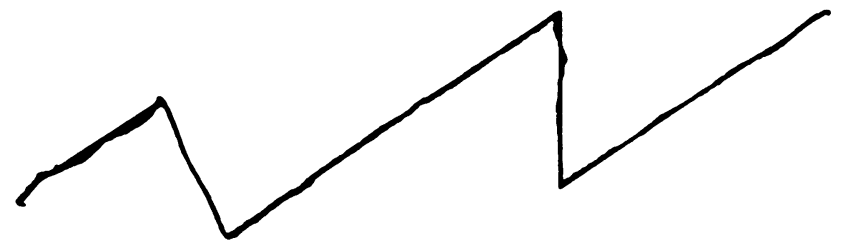

Patient 2

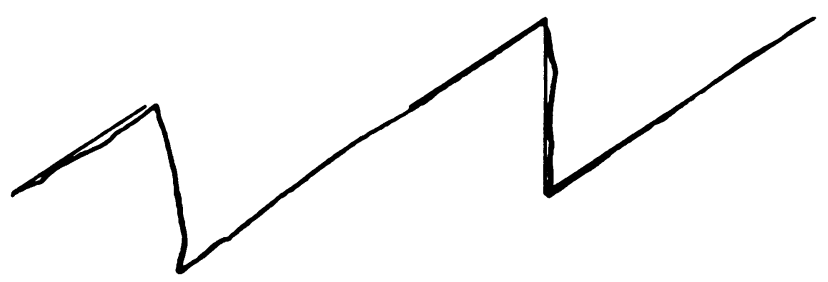

Patient 3

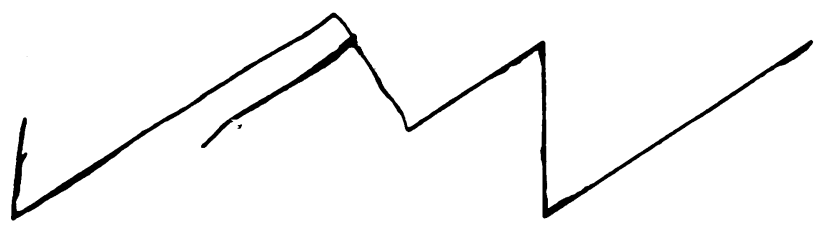

Patient 4

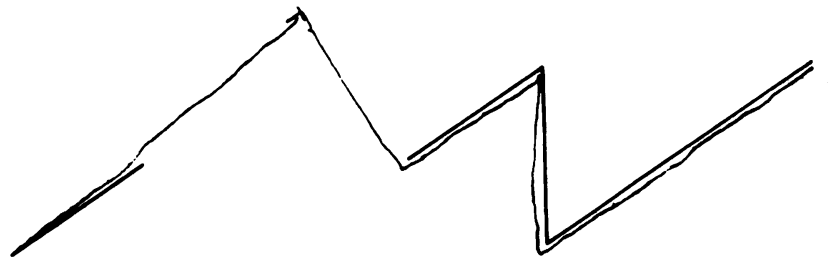

Patient 5

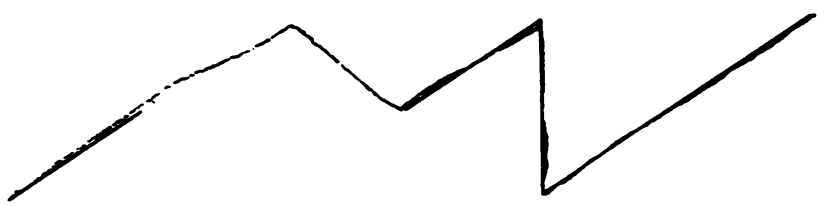

Patient 6

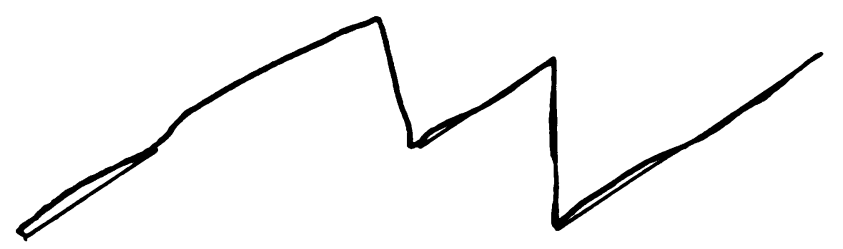

Figure 2 Samples of impaired tracing performance (form errors). correlation was found between the total score for the tracing task and the MMSE score $(r=0.54, p=0.046)$.

For controls, the MMSE score correlated positively with the Benton Line score $(r=0.62, p=0.032)$, although not with the tracing task. Performance on the Benton Line test was inversely related to error size on the directional forecast test for controls $(r=-0.58, p=0.048)$, but not for patients.

Finally, no significant correlations were found between motor severity (total extrapyramidal score on the CUPDRS) and any of the three visuospatial tests for PD patients, although a negative correlation between motor severity and the total Benton Line score approached significance $(r=-0.57, p=0.068$; motor scores were unavailable for 3 patients).

\section{Discussion}

In our experiment, PD patients were poorer at tracing a partially occluded sawtooth path than controls matched for age, education and general intellectual function. Tracing performance was similar for both groups when one angle of the sawtooth was removed. PD patients, however, made significantly more errors than controls when two consecutive angles were obscured. When tracing performance was analysed in detail, PD patients had significantly greater difficulty than controls in generating the basic form of the figure (number of turns and their direction with respect to the horizontal midline). These results confirm those found by Stern et al. ${ }^{23}$

In addition, our study demonstrates that these tracing difficulties were unlikely to arise from impaired visuoperceptual function, since the performance of patients on the Benton Line Orientation test and on the directional forecast test was no worse than that of controls. Also, no significant correlation was found between these perceptual tasks and tracing performance in patients. These tracing difficulties cannot be attributed to loss of fine motor control; patients were no worse than controls in generating the movements necessary to draw at least one complete turn (Path 2). Conversely, tracing performance in PD patients did correlate with the MMSE. We suggest that these tracing difficulties arise from a breakdown in higherorder motor control. As patients and controls were matched for general intellectual function, this impairment is selective and does not merely result from reduced global cognitive function. What is the nature of this impairment?

The ability to perform tracing tasks such as the one used here depends on at least three cognitive processes: 1) Perceptual integrity required to analyse the basic components of the stimulus to be traced and to assess the accuracy of their reproduction; 2) The development of an internal model of the stimulus to enable and 3) The translation of this model into action. These processes may be considered in relation to Kritchevsky's ${ }^{23}$ divi- 
Table 1 Point system for scoring the sawtooth tracing task.

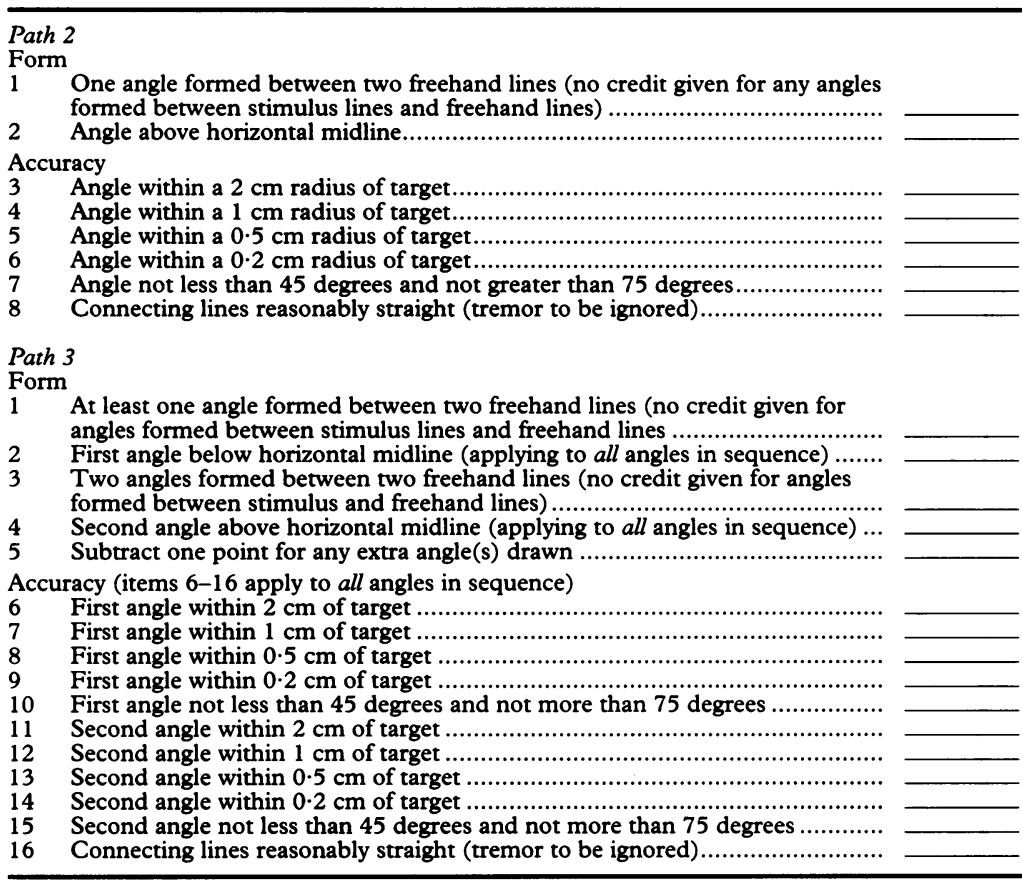

sion of visuospatial function into 5 basic categories: spatial perception (object localisation, line orientation detection and matching), spatial memory, spatial attention (to left and right hemispace), spatial mental operations (for example, mental rotation and mirrorreversal) and spatial construction. On this basis, 1) (perceptual integrity) corresponds to Kritchevsky's spatial perception; 2) (internal model) corresponds to spatial memory and 3) (translation into action) is relevant to spatial construction.

The first of these processes seems the least likely to underlie tracing difficulties in PD; our results, as well as previous studies, demonstrate that PD patients are able to judge line and angle orientation and are able to predict the intersection of converging lines. In addition, one study has shown that PD patients can identify degraded line drawings as well as normal subjects, ${ }^{24}$ implying that patients in our experiment were capable of inferring the complete shape of the tracing stimulus, even when sections of this stimulus were occluded. There is evidence that patients are impaired in visual matching, ${ }^{25-27}$ raising the possibility that they experience difficulty in judging similarities and differences

Table 2 Mean (SD) scores for each test

\begin{tabular}{|c|c|c|}
\hline Test & $P D s$ & Controls \\
\hline Benton Line & $20 \cdot 4(4 \cdot 0)$ & $19 \cdot 0(4 \cdot 1)$ \\
\hline $\begin{array}{l}\text { Directional Forecast } \\
\text { Task A } \\
\text { Task B } \\
\text { Left } \\
\text { Right }\end{array}$ & $\begin{array}{l}120 \cdot 7(45 \cdot 7) \\
391 \cdot 4(141 \cdot 3) \\
234 \cdot 9(79 \cdot 3) \\
277 \cdot 1(101 \cdot 2)\end{array}$ & $\begin{array}{l}111.5(51.7) \\
433.7(81.6) \\
256.4(61.8) \\
288.8(63.9)\end{array}$ \\
\hline $\begin{array}{r}\text { Tracing Task } \\
\text { Total score } \\
\text { Path } 2 \text { form } \\
\text { accuracy } \\
\text { total } \\
\text { Path } 3 \text { form } \\
\text { accuracy } \\
\text { total }\end{array}$ & $\begin{array}{r}11.5(3.2) \\
1.9(0.3) \\
3.4(0.8) \\
5.4(1.1) \\
3.1(1.0) \\
3.0(1.9) \\
6.1(2.7)\end{array}$ & $\begin{array}{r}13.8(4.2) \\
1.9(0.3) \\
3.7(1.6) \\
5.5(1.8) \\
3.8(0.6) \\
4.3(2.2) \\
8.2(2.4)\end{array}$ \\
\hline
\end{tabular}

between self-produced drawings and model stimuli on which they are based (whether present externally or via internal representation). However, the evidence for a visual matching deficit in PD is highly equivocal; Boller et $a l^{6}$ found no difference between patients and controls on several matching tasks where no motor output was required. Also, the figure to be traced in our experiment was relatively simple and repetitive in form. In contrast, the matching stimuli employed above ${ }^{2526}$ were complex and may have caused difficulties in switching attention between various attributes.

For memory, the second process identified above, it is possible that PD patients were unable to develop an internal model of the stimulus. If so, it may be that when external visual guidance was withheld, either declarative memory for the form of the complete figure or procedural memory for the movement sequence was insufficient to enable the occluded parts of the figure to be generated. Evidence regarding declarative visual memory in non-demented PDs is controversial, however, with some studies showing impairment $^{6112527-29}$ and others showing no difference between patients and controls. ${ }^{6242530-33}$ Some of the tests showing impairment, however, required patients to draw $^{67-29}$ (see below) and that when visual recall scores in one study were adjusted for impairment in copying ability, differences between patients and controls in the recall score were abolished. ${ }^{24}$ Over half of the patients in one study ${ }^{6}$ were also taking anticholinergic medication. The status of procedural memory in PD is also equivocal; studies have demonstrated intact pursuit rotor learning in mild and/or non-demented patients, ${ }^{34-36}$ although evidence for preserved predictive tracking of irregular movement paths is more controversial. ${ }^{37-39}$

In summary, it seems unlikely that perceptual and memory problems play a major causal role in the higher-order tracing difficulties found here and elsewhere. There are, however, compelling reasons to anticipate difficulties with process 3-the interface between visual processing and/or representation of a stimulus and translation into action. Several studies have shown that PD patients are impaired in constructional tasks that require a complete figure to be copied 324293040 (thereby eliminating dependence on visual memory). Consistent with these findings, PD patients were unable to rotate a pair of rods to match the angle of a pair they had previously inspected or palpated, ${ }^{41}$ even though several studies (and our results) have shown passive judgement of line orientation to be intact in patients. What factors might underlie this constructional difficulty?

One contributing factor may be purely sensory; previous studies show that PD patients have abnormal proprioception, ${ }^{42}{ }^{43}$ raising the possibility that they have difficulty in judging whether a motor command has been correctly executed. This would shed light on the apparent dependence shown by patients on visual 
guidance in the our experiment, as the latter presumably compensate for impaired movements resulting from abnormal afferent feedback. However, the generation of symbolic gestures, ${ }^{10}$ common motor sequences, ${ }^{27}$ simple movements (such as those required for the production of simple speech sounds) ${ }^{44}$ and the miming of object use to verbal command (that is, without visual guidance) ${ }^{10}$ are unimpaired in PD (although this is not a uniform finding), ${ }^{45}$ suggesting that overlearning may provide protection against this source of error.

A second factor is that $\mathrm{PD}$ patients are impaired in switching repetitive motor sequences, ${ }^{46}$ implying that they might have difficulty in changing direction at each angle of the sawtooth figure. This is an unlikely candidate in the present case, however, since difficulty with motor switching would impair the generation of any angle. Yet patients had no difficulty in tracing the pathway when a single angle was occluded.

Third, the role of external guidance leads to a consideration of perceptual motor dysfunction, ${ }^{23}$ that is, difficulty in coordinating motor and perceptual activities necessary to perform constructional tasks. Results from our study indicate that PD patients can use external guidance while tracing a stimulus, provided the tracing is superimposed over (that is, contiguous with) the stimulus. However, with many visuomotor tasks, the visual stimulus and the required movement are spatially disconnected and when this occurs, PD patients show difficulties. This is the case with freehand copying of a stimulus (see above), when the generation of movements or movement sequences is required in response to previous visual information (whether information is given in the form of cueing, ${ }^{44}$ symbolic representation ${ }^{8}$ or demonstration $)^{45}$ and during occlusion of a stimulus during tracing, as in our experiment. Once spatial disconnection between the visual stimulus and the required movement occurs, some degree of planning is required to translate information processed from that stimulus to movement. Here lies the probable locus of perceptual motor dysfunction. We therefore suggest that difficulties in these tasks represent a common failure in PD patients to use sensory information accurately to plan and execute complex and/or new movements.

The authors are grateful to Michael Almog and Mark Groves for scoring the directional forecast and tracing tasks employed in our experiment.

This work was supported by Federal grants AG02802, AG07232, the Parkinson's Disease Foundation (New York) and by the Center for Alzheimer's Disease Research in New York City.

1 Flowers K. Lack of prediction in the motor behaviour of Parkinsonism. Brain 1978;101:35-52.

2 Stern Y, Mayeux R, Rosen J, Ilson J. Perceptual motor dysfunction in Parkinson's disease: a deficit in sequential and predictive voluntary movem
Neurosurg Psychiatry 1983;46:145-51.

3 Stern Y, Mayeux R, Rosen J. Contribution of perceptual motor dysfunction to construction and tracing disturbances in Parkinson's disease. $\mathcal{f}$ Neurol Neurosurg Psychiatry 1984;47:983-89.

4 Stern Y. The basal ganglia and intellectual function. In: Schneider JS, Lindsey TI, eds. The basal ganglia and behavior. Toronto: Hans Huber Publishers, 1987: 169-74.

5 Danta G, Hilton $R$. Judgement of the visual vertical and horizontal in patients with parkinsonism. Neurology 1975;25:43-7

6 Boller F, Passafiume D, Keefe NC, Rogers K, Morrow L Kim Y. Visuospatial impairment in Parkinson's disease. Arch Neurol 1984;41:485-90.

7 Wasserstein J, Borod JC, Bodis-Wolner I, Golstein Y, Yahr M. Visual-perceptual deficits in Parkinson's dis-

8 Harrington DL, Haaland KY. Sequencing in Parkinson's disease. Abnormalities in programming and controlling disease. Abnormalities in programm
movement. Brain 1991;114:99-115.

9 Raskin S, Borod J, Wasserstein J, Bodis-Wolner I, Coscia L, Yahr M. Visuospatial orientation in Parkinson's dis$\mathrm{L}$, Yahr M. Visuospatial orientation
ease. Int $\mathcal{f}$ Neuroscience 1990;51:9-18.

10 Goldenberg G, Wimmer A, Auff E, Schnaberth G. Impairment of motor planning in patients with Parkinson's disease: evidence from ideomotor apraxia testing. $\mathcal{F}$ Neurol Neurosurg Psychiatry 1986;49:1266-72.

11 Levin BE, Llabre MM, Weiner WJ. Cognitive impairments associated with early Parkinson's disease. Neurology 1989;39:557-61.

12 Levin BE, Llabre MM, Reisman S, Weiner MD, SanchezRamos J, Singer C, Brown MC. Visuospatial impairment in Parkinson's disease. Neurology 1991;41:365-69.

13 Girotti F, Carella F, Grassi MP, Soliveri P, Marano R, Caraceni T. Motor and cognitive performances of Parkinsonian patients in the on and off phases of the Parkinsonian patients in the on and off phases of the
disease. $f$ Neurol Neurosurg Psychiatry $1986 ; 49: 657-60$.

14 Della Sala S, Di Lorenzo G, Giordano A, Spinnler H. Is there a specific visuo-spatial impairment in Parkinsonians? I Neurol Neurosurg Psychiatry 1986;49. 1258-65.

15 Stern MB. The clinical characteristics of Parkinson's disease and parkinsonian syndromes: diagnosis and assessment. In: Stern MB, Hurting HI, eds. The comprehensive management of Parkinson's disease. New York: PMA Publishing Corp, 1978:3-50.

16 Hoehn MM, Yahr MD. Parkinson's disease: onset, progression and mortality. Neurology 1967;17:427-442.

17 Schwab RS, England AC Jr. Projection techniques for evaluating surgery in Parkinson's disease. In: Gillingham FJ, Donaldson IM, eds. Third symposium on Gillingham FJ, Donaldson IM, eds. Third symposium on
Parkinson's disease. Edinburgh, UK: Scotland: E and S Parkinson's disease. Edinburgh,

18 Mayeux R, Chen J, Mirabello E, et al. An estimate of the incidence of dementia in idiopathic Parkinson's disease. Neurology 1981;40:1513-17.

19 Mayeux R, Stern Y, Rosen J, Leventhal J. Depression, intellectual impairment, and Parkinson's disease. Neurology 1981;31:645-50.

20 Folstein MF, Folstein SE, McHugh PR. "Mini-mental state": a practical method for grading the cognitive state of patients for the clinician. $\mathcal{F}$ Psychiatry Res 1975;12: $189-98$.

21 Stern Y, Sano M, Paulson J, Mayeux R. Modified MiniMental State Examination: validity and reliability. Neurology 1987;37(Suppl 1):179

22 Benton A, Hamsher K, Varney N, Spreen O. Contributions to neuropsychological assessment. New York, NY: Oxford to neuropsychological asses
University Press 1983.

23 Kritchevsky $M$. The elementary spatial functions of the brain. In: Stiles-Davis J, Kritchevsky M, Bellugi U, eds. Spatial cognition. Brain bases and development. Hillsdale, NJ: Lawrence Erlbaum, 1988:111-40.

24 Cooper JA, Sagar HJ, Jordan N, Harvey NS, Sullivan EV Cognitive impairment in early, untreated Parkinson's disease and its relationship to motor disability. Brain 1991;114:2095-122.

25 Sahakian BJ, Morris RG, Evenden JL, Heald A, Levy R, Philpot M, Robbins TW. A comparative study of visuospatial memory and learning in Alzheimer-type dementia and Parkinson's disease. Brain 1988;111:695-718.

26 Downes JJ, Roberts AC, Sahakian BJ, Evenden JL, Morris RG, Robbins TW. Impaired extra-dimensional shift performance in medicated and unmedicated Parkinson's disease: evidence for a specific attentional dysfunction. disease: evidence for a specific atte
Neuropsychologia 1989;27:1329-43.

27 Pirozzolo FJ, Hansch EC, Mortimer JA, Webster DD, Kuskowski MA. Dementia in Parkinson disease: an neuropsycholog
$1: 71-83$.

28 Dubois B, Pillon B, Sternic N, Lhermitte F, Agid Y. Ageinduced cognitive disturbances in Parkinson's disease. Neurology 1990;40:38-41.

29 Richards M, Stern Y, Sano M, Mayeux R. Patterns of neuropsychological impairment are distinct in Parkinson's disease, Alzheimer's disease and Parkinson's disease dementia. Neurology 1991;41(Suppl 1): 177

30 Stern Y, Sano M, Mayeux R. Comparisons of dementia and intellectual change in Parkinson's and Alzheimer's disease. f Clin Exp Neuropsychol 1987;9:66 (Abstr).

31 Sullivan EV, Sagar HJ, Gabrieli JDE, Corkin S, Growdon JH. Different cognitive profiles on standard behavioral tests in Parkinson's disease and Alzheimer's disease. $\mathcal{f}$ tests in Parkinson's disease and Alzheimer
Clin Exp Neuropsychol 1989;11:799-820.

32 Flowers KA, Pearce I, Pearce JMS. Recognition memory in Parkinson's disease. $\mathcal{f}$ Neurol Neurosurg Psychiatry

33 Taylor AE, Saint-Cyr JA, Lang AE. Frontal lobe dysfunction in Parkinson's disease. Brain 1986;109:845-83. 
34 Heindel WC, Salmon DP, Shults CW, Walicke PA, Butters N. Neuropsychological evidence for multiple implicit memory systems: a comparison of Alzheimer's Huntington's, and Parkinson's disease patients. $\mathscr{f}$ Neuroscience 1989;9:582-87.

35 Harrington DL, Haaland KY, Yeo RA, Marder E. Procedural memory in Parkinson's disease: impaired motor but not visuoperceptual learning. $\mathcal{f}$ Clin Exp Neuropsychol 1990;12:323-39.

36 Bloxham CA, Mindel LA, Frith CD. Initiation and execution of predictable and unpredictable movements in Parkinson's disease. Brain 1984;107:371-84.

37 Day BL, Dick JPR, Marsden CD. Patients with Parkinson's disease can employ a predictive motor stratParkinson's disease can employ a predictive motor strat-

38 Stern Y. Response to Day, et al. $\mathcal{f}$ Neurol Neurosurg Psychiatry 1986;49:107

39 Frith CD, Bloxham CA, Carpenter KN. Impairments in the learning of a new manual skill in patients with Parkinson's disease. $f$ Neurol Neurosurg Psychiatry 1986;49:661-8.

40 Villardita C, Smirni P, Le Pira F, Zappala G, Nicoletti F.
Mental deterioration, visuoperceptive disabilities and constructional apraxia in Parkinson's disease. Acto Neurol Scand 1982;66:112-120.

41 Hovestadt A, de Jong GJ, Meerwaldt JD. Spatial disorientation as an early symptom of Parkinson's disease. Neurology 1987;37:485-7.

42 Marsden CD. The mysterious motor function of the basal ganglia: the Robert Wartenberg lecture. Neurology 1982;32:514-39.

43 Markham CH. The "motor" vs. "sensory" enigma in Parkinson's disease. In: Schneider JS, Lidsky TI, eds. Basal ganglia and behavior: sensory aspects of motor functioning. Toronto: Hans Huber, 1985:161-7.

44 Connor NP, Abbs JH. Task-dependent variations in Parkinsonian motor impairments. Brain 1991;114: Parkinsoniar

45 Sharpe MH, Cermak SA, Sax DS. Motor planning in Parkinson patients. Neuropsychologia 1983;21:455-62.

46 Cools AR, Van Den Bercken JHL, Horstink MWI, Van Spaendonck KPM, Berger HJC. Cognitive and moto shifting aptitude disorder in Parkinson's disease. $f$ Neurol Neurosurg Psychiatry 1984;47:443-53. 